\title{
Towards Smart City Energy Analytics: Identification of Consumption Patterns Based on the Clustering of Daily Electric Consumption Curves
}

\author{
Fateh Nassim Melzi, Mohamed Haykel Zayani, Amira Benhamida, \\ François Stephan, Allou Same and Latifa Oukhellou
}

\begin{abstract}
This paper presents the application of clustering algorithms to daily energy consumption curves of buildings. Our aim is to identify a reduced set of consumption patterns for a tertiary building during one year. These patterns depend on the temperature throughout the year as well as the type of the day (working day, work-free day and school holidays). Two clustering approaches are used independently, namely the K-means algorithm and the Expectation-Maximization algorithm based on Gaussian Mixture Model (EM-GMM). The clustering results obtained with the two algorithms are analyzed and compared. This study represents the first step towards the development of a prediction model for energy consumption.
\end{abstract}

F.N. Melzi $(\bowtie) \cdot$ M.H. Zayani · A. Benhamida · F. Stephan IRT SystemX, 8, Avenue de la Vauve, 92400 Palaiseau, France e-mail: nassim.melzi@irt-systemx.fr

M.H. Zayani

e-mail: mohamed.zayani@irt-systemx.fr
A. Benhamida
e-mail: amira.benhamida@irt-systemx.fr
F. Stephan
e-mail: francois.stephan@irt-systemx.fr

F.N. Melzi · A. Same $\cdot$ L. Oukhellou

IFSTTAR, 14-20 Boulevard Newton Cité Descartes Champs sur Marrne, F-77447 Marne la

Vallée, France

e-mail: allou.same@ifsttar.fr

L. Oukhellou

e-mail: latifa.oukhellou@ifsttar.fr 